Amazonía Peruana, Tomo XII № 24, 1994; pp. $105-123$

\title{
TRANSFORMACIONES EN LA
}

\section{AMAZONÍA \\ Estatus, Genero y Cambio \\ ENTRE LOS ASHÁNINKA}

JUDY TIZÓN

Los efectos negativos sobre los indígenas amazónicos de fuerzas exteriormente controladas tales como la construcción de carreteras, desforestación y colonización han sido denunciados por investigadores y la prensa popular, pero no se ha presentado atención a las modificaciones en las relaciones de género y el estatus (posición social) de la mujer como resultado de este proceso de transformación cultural. Se examina este proceso entre los Asháninka del bosque tropical húmedo del Perú durante los últimos veinte años, con respecto a los estudios relevantes y los acontecimientos políticos regionales.

The negative effects upon indigenous amazonians of externally controlled forces such as road construction, deforestation and colonization have been widely reported by scholars and the popular press, but little atention has been paid to the alterations in gender relations and women's status brought about during this process of sociocultural transformation. This process is examined among the Ashaninka of the central Peruvian rainforest over the past twenty years, whith reference to relevant studies and regional political developments. 
Los años recientes han visto un incremento dramático de la atención, tanto de estudiosos como pública, focalizada en las difíciles condiciones de la Selva Amazónica y sugente. Los efectos negativos derivados de la injerencia de fuerzas externas tales como la construcción de caminos, deforestación y colonización de culturas indígenas han sido largamente denunciadas, pero poca atención ha sido prestada a las al teraciones de las relaciones entre géneros y al estatus de la mujer a medida que pequeñas sociedades hortícolas sin clases ni mercado se insertan en el sistema socio-económico de clases nacional. El propósito de esta monografía es explorar las condiciones que han llevado a un incremento de la subordinación de las mujeres dentro de un grupo de esta índole, los Asháninka de la cuenca del río Pichis de la Selva Central Peruana ${ }^{(1)}$, y comparar la situación Asháninka con la de muchos otros grupos de la selva tropical peruana descrita en estudios relevantes.

Los Asháninka descritos en este estudio pertenecen al grupo lingüístico Arawak, practican la horticultura, la pesca, la caza (en un menor grado), y viven a lo largo de las cabeceras del Río Pichis y sus afluentes.

En las economías domésticas como las de subsistencia horticultural descentralizada, el estatus de la mujer debe ser visto más bien como situacionalmente dependiente antes que uniformemente subordinado al del hombre. Los indicadores de la autonomía femenina (y relativa ausencia de estratificación sexual) incluirían la libertad de las mujeres para disponer de los productos de su labor y para tomar decisiones respecto a sus actividades tan independientemente como los hombres (si y cuándo viajar para visitar parientes, ir a pescar, y qué comprar o vender). Tener capacidad de veto respecto a los esfuerzos conjuntos de esposo y esposa y la capacidad para influir en las actividades y el comportamiento de los hombres por el otorgamiento o la retención de bienes valorados o servicios son otros ejemplos del control de las mujeres sobre sus propias vidas. La emergencia de la subordinación femenina, por otra parte, es indicada cuando las mujeres se hacen económicamente más dependientes del hombre que viceversa, son sujetos de abuso físico por parte de los hombres, cuando ellas pierden el derecho de disponer de los productos de su trabajo, y cuando se convierten en objeto de las decisiones de sus esposos quienes deciden dóndevivir, qué compraro producir, y cuándo viajar ${ }^{(2)}$. En suma, las mujeres pierden prestigio cuando ellas pierden la propiedad y el control de recursos valiosos.

Sugiero que tres factores conjuntos contribuyen a la erosión de la autonomía de las mujeres y a la emergencia de la subordinación generalizada de las 
mujeres (como grupo) a medida que estas sociedades no estratificadas se ven envueltas en la economía de mercado nacional. Primero; las manufacturas femeninas tradicionales no son valoradas en el mercado exterior, y las mujeres dejan de producir aquellas que son valoradas internamente porque sustitutos industriales baratos son rápidamente asequibles. Segundo, el sesgo masculino de las plazas del mercado moderno brinda a los indígenas de un acceso preferente al dinero, haciéndolos compradores de los nuevos artículos que reemplazan las manufacturas de las mujeres; $y$, finalmente, la inducción del mercado a un cambio que va de una organización más comunal social ( y de trabajo) a economías domésticas nucleares independientes incrementa el aislamiento doméstico cuya resultante para las mujeres es más trabajo y menos control sobre los resultados de la producción doméstica. Así, el trabajo de las mujeres recibe menor recompensa que el de los hombres, en la medida en que la recompensa viene a ser definida como dinero, las mujeres pierden prestigio a medida que abandonan el control de fuentes de valor, y pierden poder conforme comienzan a depender de los hombres, quienes son los productores primarios en la economía transformada.

Aun si está bajo la severa presión del desarrollo, la vasta selva de la Amazonía tropical representa una de las pocas regiones que quedan inexplotadas en el mundo. Como tal, contiene algunas de las únicas culturas indígenas todavía en los estadios tempranos de transformación por las fuerzas del sistema del mercado mundial. Mi investigación allí abarca el período 1968-1988, un lapso de esporádica pero cresciente colonización y actividad del mercado, en el cual he logrado observar los primeros efectos de estas fuerzas sobre los Asháninka. Mientras quẻ aparecen patrones generales de la comparación de grupos de la Amazonía similares en la medida en que son afectados por la política nacional y las fuerzas económicas, no puede asumirse que todos los horticultoristas amazónicos exhiben sistemas de relaciones de géneros similares. Por otra parte, la prominencia del Yanamamo en las discusiones de las relaciones masculino-femenina ( Harris 1983, Shapiro 1972) desmiente su unicidad; en un continuum desde la extrema estratificación sexual a la paridad sexual, ellos representan lo primero. Un grupo todavía bajo dominación masculina aunque a un grado menos extremo es el Sharanahua tal como lo describe Siskind (1973) en el cual las mujeres dependen de los hombres a causa del recurso más grave, el alimento. Los Mundurucu, (Murphy \& Murphy 1974), por otro lado, exhiben una autonomía económica femenina pero comparten con los otros dos grupos una ideología de superioridad masculina y antagonismo sexual. Los Asháninka y otros grupos aquí discutidos representan el otro extremo del continuum. Varían en el estatus y la autonomía acordados a las 
mujeres debido al grado y particulares circunstancias de inserción en el mercado. Tradicionalmente, exhiben un grado relativamente alto de paridad sexual (de recompensa, prestigio, y poder) y relativa armonía en las relaciones de género.

Los estimados del total de la población Asháninka varían entre 25,000 (Devenan 1974:93) y 45,000 (Chirif 1981: 25), haciendo de ellos uno de los grupos más extensos de los que se encuentran en la Amazonía. La población indígena del área de estudio es entre 8,000 y 10,000 , aproximadamente $60 \%$ de la población total de esa región, pero cercana aún más al $90 \%$ de la población exterior al pueblo de Puerto Bermudez (capital de distrito y único pueblo de la región) y sus alrededores inmediatos (Tizon 1988).

Un asentamiento típico consiste en un agrupamiento (3-15) de casas de postes y techos de paja, las más de las veces de plataformas abiertas, unas cuantas con paredes, ubicadas en una margen alta a corta distancia del río. El área inmediatamente alrededor de las estructuras se mantiene despejada de toda vegetación como impedimento para la aproximación de serpientes u otros seres indeseables. Pequeños huertos de trigo, habas, plátanos y otras cosechas de estación son cultivadas a lo largo de las márgenes del río durante la estación seca; parcelas más largas de mandioca y árboles frutales surtidos y otras cosechas se extiende tierra adentro. Quién y cuántas personas hay en las residencias varía grandemente; llegan visitantes, los residentes salen a visitar a otros, los hombres con o sin sus familias salen periódicamente marchan a trabajar por jornales en las plantaciones río abajo o fuera del área. En un día típico, habrá unas cuantas personas en el recinto; la mayoría marchará a sus huertos por buena parte del día (aunque solamente parte del tiempo en realidad seconsume en faenas en el huerto), retornando a finales de la tarde para bañarse, comer y socializarse. Otras actividades típicas pero no diarias incluyen tejer ropa, cestas, y esteras (principalmente por las mujeres), pescar (ambos sexos) y fabricar flechas (los hombres). En las noches claras, iluminadas por la luna, hay baile, cantos, se cuentan historias y se tocan instrumentos. Subjetivamente, los Asháninka dan la impresión a los extraños de ser hospitalarios, dispendiosos, felices y calmados.

Históricamente, el grupo ha tenido contactos intermitentes con el mundo externo. La colonización permanente de algunas de las áreas tradicionales de habitación en años recientes ha devenido en el establecimiento de comunidades permanentes de fuereños. Debido a la naturaleza del medio físico y a la tradicional adaptación Asháninka a él, la reacción más común a los contactos 
abusivos consumados ha sido retirarse de las áreas de contacto. Poco a poco este patrón de respuesta ha cambiado como resultado del continuo influjo de los colonizadores.

Los Asháninka aún podrían ser descritos comosemi-sedentarios aunque las familias en ésta área se desplazan menos frecuentemente que en el pasado. Se hallan envueltos en variados grados en el sistema del mercado local, pero casi toda familia es autosuficiente en términos de comida y vivienda. Adaptados sólo parcialmente a la economía de mercado, no han abandonado todavía los patrones horticolas efectivos y tradicionales de la selva tropical. Indudablemente, el empleo de utensilios de metal ha alterado significativamente la cantidad de tierra que puede ser abierta y mantenida, y las cosechas de hoy en día muestran una mucho mayor variedad (posiblemente sustituyéndolas por aquellas que antes proveían al forraje). Muchos rasgos salientes del sistema social tradicional han sido mantenidos: la independencia de cada familia, la ausencia de autoridad política centralizada, el énfasis ideológico en el igualitarismo, y la importancia del parentesco y la reciprocidad.

Concernientes a la discusión respecto a los roles de las mujeres entre los Asháninka existen otros dos estudios de grupos hortícolas de la amazonía peruana cercanos. El primero es un análisis comparativo de Kathleen y Anthony Stocks (1984) del relativo involucramiento en el mercado de los Candoshi, los Cocamilla y los Shipibo y sus relaciones con el estatus de las mujeres. El segundo es la descripción de roles sexuales entre los Machiguenga no aculturados, un grupo lingüística y culturalmente bastante similar a los Asháninka, debido a Orna y Allen Johnson (1975). La utilización de estos tres estudios separados permite una generalización de antrecruzamiento cultural con una mínima reconstrucción especulativa.

En su comparación de las mujeres Candoshi, Cocamilla y Shipibo, los Stocks (1984) concluyen que las fuerzas del mercado crean una pérdida de autonomía, haciendo de las mujeres "simples miembros de una fuerza de trabajo coordinada y controlada por grupos de hombres" (Stocks 1984: 68, traducción mía). En su perspectiva, el proceso es definido como una disminución en la complejidad del trabajo (es decir, en la interdependencia basada en la división del trabajo entre hombres y mujeres), la pérdida del control de las mujeres de la producción y la distribución de recursos, y un aumento en la segregación social de mujeres y hombres (Stocks 1984:68). En otras palabras, el mercado causa un movimiento desde estatus más igualitarios, definidos sobre bases distintas al sexo, a una estratificación sexual en la que podríamos hablar 
del "estatus" de la mujer (definido en el fundamento del sexo) comogeneralmente subordinado al del hombre.

Los Stocks señalan dos factores que consideran de primera importancia en lo que respecta a la posición de las mujeres en estos grupos hortícolas conforme se ven envueltos en la economía de mercado: primero, el nivel de aculturación, de la sociedad cuando entra en contacto con un mercado monetario $y$, en segundo lugar, el nivel de desarrollo del mercado regional o nacional, en términos de sus patrones de distribución y el valor asignado por los consumidores de fuera a los bienes producidos por las mujeres (1984:67). Desafortunadamente para las mujeres de la Amazonía este valor es usualmente bajo.

De los tres grupos comparados ellos encuentran que las mujeres Candoshi disfrutan de un al to grado de independencia y autonomía debida a la inserción limitada en el mercado del grupo y al control de las mujeres sobre los productos valorizados internamente (masato o cerveza de mandioca) y externamente (harina de mandioca o farina). Los hombres despejan los huertos, pero cada sexo es responsable de plantar y cosechar cosechas particulares; ambos cultivan. Las mujeres mantienen cerca del total del control de la cosecha más importante, mandioca, tanto en forma de productos alimenticios como en la forma de masato que tiene un rol muy prominente en todas las actividades sociales; solamente las mujeres preparan y distribuyen el masato. La relativamente pequeña involucración en el mercado que existe, consiste en que los hombres venden una piel de animal o un grupo, o en que las mujeres venden harina de mandioca (farina) o animales domés ticos; los hombres aparentemente conducen la venta de los productos de las mujeres, pero la productora dispone del ingreso como ella vea conveniente (Stocks 1984:69).

Entre los Cocamilla orientados al mercado, en contraste, las mujeres ocupan una posición claramente subordinada a los hombres; mientras ellas producen bienes, los hombres controlan sus distribución y hay una pequeña complementariedad en la división del trabajo. Los Cocamilla han tenido la historia más larga de contactos con el exterior y están completamente insertados en la agricultura comercial y la pesca (Stocks 1984: 70). Tanto hombres como mujeres están activamente comprometidos en la horticultura y el tratamiento de todas las cosechas, pero son los hombres quienes comercializan lo producido y controlan los ingresos. Las mujeres son responsables de la crianza de animales domésticos y de preparar masato; sin embargo, a diferencia de los Candoshi, los ingresos familiares procedentes de todas las ventas son ad- 
ministrados por el esposo y, tal como las mujeres, los hombres sirven el masato. Los hombres toman todas las decisiones económicas y controlan todos los ingresos en efectivo de la familia. Los Stocks describen a las mujeres Cocamilla como claramente inferiores en la vida ritual y social, las actividades de las que están espacialmente segregadas en tanto que sexo; las mujeres raramente expresan opiniones en compañía mixta (Stocks 1984: 71).

Una excepción a este patrón de subordinación en aumento es el alto estatus de las mujeres Shipibo a pesar de una fuerte inserción en el mercado. Como los Cocamilla, los Shipibo también parecen haber perdido la división sexual del trabajo en la agricultura; ambos sexos plantan, cultivan, cosechan y procesan la cosecha para el mercado. Las cosechas de mayor valor, como el yute, son vendidas al Banco Agrario Nacional y los hombres controlan los ingresos; a diferencia de los Cocamilla, los jornales del trabajo de la recolección son retenidos para el trabajador, sea hombre o mujer (Stocks 1984:72). La importancia del masato es todavía menor en tre los Shipibo: según cabe preśumir, las mujeres lo producen (los Stocks no lo dicen específicamente), pero es servido por ambos sexos y ha sido reemplazado largamente por la cerveza comercial (Stocks 1984:73). El alto grado de autonomía y control de las mujeres Shipibo está directamente relacionado al hecho de que ellas son las productoras exclusivas y distribuidoras de productos altamente valorados en el mercado urbano externo: cerámica de los nativos, joyería, y textilería decorada. Los Stocks vinculan esto con el que los Shipibo tuviesen su ingreso en el sistema del mercado en una época en la que los consumidores urbanos apreciaban las artesanías indígenas (Stocks 1984:74). Yo añadiría que también podría ser el caso que estas artesanías tradicionales Shipibo fuesen más elaboradas que aquellas encontradas en otras culturas amazónicas, y por ello más atrayentes para los consumidores urbanos.

Las comparaciones efectuadas arriba sugieren que la influencia de una economía de mercado en lo relativo a la posición de la mujer en tales sociedades se dirige a disminuirlas; en todos los casos los hombres asumieron el control de los ingresos de la venta de los productos agrícolas, y un producto tradicional de las mujeres altamente valorado, el masato, disminuyó en importancia dentro de la cultura y las mujeres perdieron los derechos de distribución exclusiva. Solamente en el caso de los Shipibo, donde un producto exclusivamente femenino encontró favor en el mercado externo, las mujeres fueron capaces de mantener un grado de independencia económica y autonomía. Aunque los Stocks no especulan en torno a su importancia, es interesante notar que el patrón de residencia para los Candoshi y los Shipibo es matrilocal y para los Cocamilla, patrilocal. 
Mi investigación entre los Asháninka sugiere que el caso de los Shipibo es altamente improbable que ocurra en otra parte. Mi presunción es que los grupos adoptan inicial y más rápidamente tecnologías nuevas que proveen ahorro y energía dentro del estilo de vida tradicional. No debería ser sorpresa el encontrar que estas mujeres abandonan de buena gana sus actividades tradicionales en las que invertian más tiempo, manufactura de ropa y alfarería, seguidas por la producción de harina de mandioca y cerveza en algunas áreas, en favor de reemplazos industriales prontamente disponibles. Después de las hachas y los machetes de acero (empleados en todas partes por la misma razón) los más ubicuos bienes industriales que se encuentran en estas sociedades son ollas de metal y telas; hasta entre Asháninka aislados los primeros tres artículos son ahora irremplazables por tecnología nativa y el último está desapareciendo rápidamente. El resultado inicial de estas sustituciones es una reducción en la carga de trabajo de las mujeres. Con todo, aun cuando el mercado aligera a las mujeres indígenas de la carga del tiempo que toman las manufacturas, las ubica en una posición dependiente respecto a los hombres, y' la carga de trabajo aligerada no es sino un estado temporal. Inevitablemente, la reducción en la base de recursos debida a presiones externas (en las tierras $\mathrm{y} / \mathrm{o}$ en el valor de mercado del trabajo o de las cosechas), 0 una elevación en el precio de susbtitutos industriales, cambiarán el equilibrio hacia más trabajo en el huerto para las mujeres en la medida en que intenten incrementar la producción de cosechas para venta (además de la subsistencia) para adquirir tales bienes. Este proceso además disminuye el tiempo disponible para las manufacturas caseras y la espiral hacia una mayor confianza en los bienes del mercado como reemplazos se vuelve firmemente establecida.

En la mayoría de las sociedades hortícolas amazónicas las mujeres contribuyen con una cantidad significativa de trabajo en la agricultura; por consiguiente, debemos examinar por qué es que los hombres han llegado a controlar la distribución de lo producido para el mercado. Parte de la respuesta yace en la dominación masculina del mercado exterior. Los compradores de sexo masculino están más a gusto negociando con vendedores de sexo masculino, los empleadores varones sólo contratarán trabajadores, los préstamos agrícolas son hechos solamente a los "cabeza de familia" quienes legalmente son solamente hombres, y los títulos de tierras se los dan sólo a los hombres. Las mujeres y los niños son considerados legalmente dependientes (Rogers 1980; Stocks 1984). Además, comerciar en el Amazonas frecuentemente implica largos viajes y ausentarse del hogar. Ahí donde esto ha sido un tradicional patrón masculino (cazar, o viajes de incursión o de comercio) y/o 
donde las mujeres han sido horticulturalistas importantes para la subsistencia podríamos esperar que sean los hombres, más bien que las mujeres, quienes asumiesen esta nueva actividad. Tal como lo destacan los Stocks (1984:75), muchas comunidades indigenas pueden funcionar por días o semanas sin los hombres pero no sin las mujeres; añade fuerza a este argumento el hecho de que las mujeres figuren prominentemente en los mercados urbanos en las regiones en las que la distancia de la casa al mercado es corta.

Los Johnsons (1975), en su artículo sobre los Machiguenga, sugieren que la organización social del trabajo es un determinante mayor de la relativa posición de hombres y mujeres. Además dividen esta variable entre tres componentes: la composición sexual del grupo de trabajo, la calidad de las relaciones interpersonales en el trabajo, y los vínculos sociales que se desarrollan a través del proceso de trabajo (Johnson 1975:635). Los grupos de trabajo colectivos ayudan a las mujeres a mantener una independencia social y una base económica, pero donde tanto hombres y mujeres están exclusivamente involucrados en grupos de producción segregados sexualmente puede resultar un antagonismo entre hombres y mujeres. Donde existe trabajo complementario y recíproco entre mujeres y hombres y las faenas son realizadas por esposo y esposa juntos, un "respeto y una mutualidad entre los sexos" es favorecida (Johnson 1975:646). Los Johnsons concluyen que las Machiguenga poseen un igualitarismo sexual más grande que cualquier otro grupo amazónico porque la organización del trabajo provee una base para la solidaridad femenina y la cooperación esposa-esposo en la forma de trabajo de la tierra en común, forrajear, viajes de pesca y manufacturas en el hogar (1975:644). Implícito en este argumento está que hay una pequeña base para la formación de la solidaridad masculina en la organización del trabajo; sugieren como posibles razones para esta ausencia de guerra, las redes políticas, y la carestía de caza lo que provoca la preclusión de la formación de partidas de caza de los hombres. (Johnson 1975:637, 639).

En un continuum que va de la menor a la mayor inserción en el mercado, los Asháninka caen entre los Machiguenga y los Candoshi y los Cocamilla y los Shipibo por otro. Al igual que los Mashiguenga y los Candoshi, permanecen como primordialmente horticulturalista en lo que atañe a su subsistencia, la autonomía femenina es relativamente alta, y las relaciones de género están colocadas en variable situación. Los patrones de trabajo de los Asháninka son similares en muchos sentidos a los de los Machiguenga: las mujeres trabajan con otras mujeres o con sus esposos; los hombres trabajan más frecuentemente solos o con sus esposas; y la mayor parte del trabajo es acompañado por 
conversación frecuente y socialización. Observo que las interacciones personales entre esposos y esposas tienden a ser amistosas; las conversaciones grupales involucran a mujeres y hombres por igual y es rara la denuncia de abuso físico sobre una mujer cometido por un hombre (y está asociado con la ebriedad cuando ocurre). En efecto, la razón de peso dada por los Asháninka para no casarse con colonos de las zonas altas era la de la reciente reputación de violencia doméstica. Tradicionalmente, y presente en algún grado, los hombres van a vivir con la familia de la esposa tras el matrimonio para realizar servicios para la desposada. La pareja suele permanecer seguido en las proximidades si la tierra está disponible; de modo que existe (como entre los Machiguenga, los Shipibo y los Candoshi) una tendencia matrilocal que estimula el trabajo conjunto femenino.

Los Asháninka son únicos entre estos grupos, en que sólo ellos representan el estadio temprano del involucramiento en el mercado en el cual la adopción de unos cuantos bienes industriales ha reducido la carga de trabajo para la subsistencia más de lo que la necesidad de adquirir dinero la ha incrementado. La vida para los Asháninka, así como para los Machiguenga, aún es relativamente fácil. Allen Johnson (1978:52) estima aproximadamente en seis las horas de ocio (aparte de las de sueño) de los Machiguenga, mi estimado para los Asháninka del Pichis es ligeramente más alto, particularmente si la estación lluviosa se incluye, ya que es cuando menos faenas agrícolas tienen lugar. Altamente involucrados en la producción para el mercado, los Shipibo y Cocamilla exceden seguramente la carga de trabajo de los Asháninka (aunque los Stocks no proveen datos cuantitativos sobre esto) mientras que el procesamiento de fariña (harina de mandioca) de los Candoshi es también una práctica cuyo consumo de tiempo no es compartida por las mujeres Asháninka. En comparación a los patrones Machiguenga, los hombres Asháninka permanecen menos tiempo y las mujeres más en labores de tierras. La diferencia más significativa está en la mayor cantidad de tiempo que las mujeres Machiguenga dedican a manufacturas domésticas y a la preparación de la comida. La relativa poca importancia entre los Asháninka, del consumo de cerveza de mandioca explica aproximadamente todas las diferencias en la producción de alimentos (volveré sobre este punto más adelante). Sin embargo, ya se han puesto en marcha en la régión del Pichis las fuerzas del mercado que pueden llevar a la pérdida de la autonomía femenina, el control, y el prestigio discutidos arriba.

Si las observaciones de los Stocks son correctas, la reducción en la complementariedad de la división del trabajo entre los sexos es indicadora de 
una erosión del estatus de las mujeres. Tradicionalmente, los hombres y las mujeres Asháninka contribuyeron casi igualmente a la subsistencia, a medida que los hombres cazaban y despejaban la selva para nuevos huertos las mujeres hacían mucho del cultivo y la recolección. Mientras que la familia extendida (la familia nuclear y los parientes agrupados) es todavía la unidad económica primaria, no hay una estricta división del trabajo en las actividades de subsistencia (con la excepción de desmontar, quemar, y la caza ocasional como actividades masculinas). Ambos sexos plantan, cultivan, cosechan y pescan.

El énfasis de los Johnsons en la importancia de la organización del trabajo para el estatus de la mujer sugiere que la necesidad de dinero en efectivo, aun cuando mínima, tiene importantes consecuencias para la cantidad de tiempo que mujeres y hombres pasan juntos. Las razones tradicionales para la separación de los hombres de sus familias -guerras de corto plazo, incursiones, y expediciones de comercio formalizadas-han sido reemplazadas por trabajo jornalero en plantaciones distantes. Trabajar por jornales todavía es solamente una actividad ocasional para los hombres, pero se halla en aumento, reduciendo el tiempo que una familia permanece junta en trabajo de horticultura, haciendo manufacturas o en esparcimiento. El trabajo asalariado introduce también la separación no tradicional del trabajo en su dimensión de interacción social. Las oportunidades de conseguir jornales (en labores como cortar árboles, cosechar café o fruta, o limpiar tierras para plantar) son acordes a la estación y solamente para hombres, y usualmente los hombres abandonan el área. Todos los varones Asháninka interrogados dijeron que preferirían llevar a sus familias durante esos viajes; por cierto, muchos de ellos lo hicieron así en un inicio y encontraron que los costos de hospedaje y alimentación allá lejos eran caros y los efectos de la ausencia del hogar desastrosos para sus huertos. En contraste, las mujeres entrevistadas dijeron que preferían permanecer en sus hogares, puesto que otras mujeres de la familia extensiva y de la comunidad no solamente provéen una cerrada red social sino también grupos de trabajo compartido para cuidar a los niños y para la horticultura. Un indicador del aumento del control masculino como algo vinculado al grado de inserción en el mercado es el hecho de que los hombres que con mayor frecuencia se comprometen en trabajo asalariado llevan consigo a sus familias; la preferencia del esposo tiene preeminencia sobre la de su esposa.

Durante los inicios de 1980, al área local experimentó un surgimiento de la actividad mercantil y la colonización estimulada por la construcción de la ruta que unía Puerto Bermudez con la carretera central a Lima y a la costa; la ruta fue inaugurada en 1984 . Esta ha brindado a los Asháninka la alternativa 
de vender su producción o trabajar localmente, aliviando la necesidad de viajar largas distancias por extensos períodos de tiempo. Como los Cocamilla y los Shipibo, sin embargo, esta actividad está dominada por los hombres; son ellos quienes efectúan las mayoría de las ventas y compras de cosechas. Los medios preferidos de obtener efectivo entre los Asháninka: Actividades empresariales a pequeña escala tales como conducir pasajeros en el río, venta ambulatoria de pequeños artículos adquiridos en el pueblo, la administración de pequeña tienda en la propia casa de uno, o sirviendo de contratistas de grupos de trabajo agrícola para los colonos, todas son ante todo actividades masculinas. La práctica de los contratistas en la que las esposas sirven como cocineras sin paga para el grupo de trabajo, evoca la práctica de los Cocamilla en la que los hombres reciben todos los ingresos del trabajo, conjunto de esposo y esposa.

A diferencia de los Shipibo, las mujeres Asháninka tienen pocas manufacturas nativas que sean posibles de encontrar favor entre los consumidores urbanos; la manufactura de cerámica ha desaparecido hace tiempo y los artículos de ropa y domésticos tienden a ser utilitarios antes que decorativos. Sin embargo, las mujeres ocasionalmente venden cestas o tejidos cuando la oportunidad se presenta por sí sola, así como animales domésticos (las más de las veces pollos, o un cerdo ocasionalmente) y productos en pequeñas cantidades. Unas cuantas mujeres aun mantienen vigente un convenio con'las familias de los colonos para la venta de productos que se hallen a disposición. Al igual que la Machiguenga y la Candoshi, la vendedora Asháninka dispone de las ganancias como considera pertinente; tradicionalmente los hombres no son dueños de aquellos productos que derivan del trabajo de sus esposas tales como ropa, cestas, o los animales que éstas han criado. Entre los Asháninka menos aculturados, mis pedidos de compra de artículos locales fueron remitidas al productor, varón o mujer, con quien tuve que pactar directamente. En áreas menos remotas de mayor inserción en el comercio, frecuentemente los hombres efectuaron la transacción (y recibieron el pago) por venta de todos los artículos. Los hombres claramente controlan la parte del león de los ingresos familiares, sin embargo mínima, puesto que ellos son los asalariados primarios. Si bien los esposos usualmente satisfacen las demandas de sus esposas de pequeños artículos, el hecho de que ellos puedan rehusarse indica la pérdida de control sobre la propiedad familiar por parte de las mujeres, tanto en la adquisición como en la disposición cuando existe una mayor dependencia del mercado.

Aunque tradicionalmente descentralizada, la estructura política impuesta por el gobierno a los Asháninka evidencia un sesgo masculino similar a aquel 
de los centros de comercio. La nueva organización política indigena requerida por la Ley de Comunidades Indígenas (D.L. 20653) par su reconocimiento oficial es encabezada por hombres que deben llegar a acuerdos con otros hombres en los gobiernos locales y nacionales ${ }^{(3)}$. Además, el gobierno interrumpió la titulación de las tierras delas comunas en favor de títulos individuales (Smith 1982:81) que por un tiempo fueron otorgados a los varones cabezas de familia con esposas e hijos legalmente definidos como dependientes. La asistencia técnica y los préstamos del gobierno para la agricultura, cuando los hubieron disponibles para la población indígena, solamente fueron otorgados a aquellos que poseian título de su tierra.

La mayor parte de la tierra de los Asháninka se halla ahora bajo los títulos provistos a través de la Ley de Comunidades Indígenas y es administrada por la ANAP (Asociación de los Asháninka del Valle del Pichis o Apatyawaka Nampitisi Asháninka Pichis), la cual es una organización legal, oficialmente reconocida que representa a 52 asentamientos en la región del Valle del Pichis. La tierra es mantenida comunitariamente por la organización y no puede ser vendida o disponerse de ella de algún otro modo. La ANAP ha elegido funcionarios y cada asentamiento tiene el suyo; las asambleas generales se llevan a cabo dos veces al año a no ser que haya problemas urgentes que discutir.

Bajo la nueva forma de organización, las mujeres asisten a las asambleas (y se les requiere que lo hagan, asi como a los hombres), pero ningún representante es mujer. Algunas mujeres desempeñan nuevos roles como los de promotoras de salud comunitaria o maestras de escuela, pero son la minoría. La falta de delegadas impide que los intereses de las mujeres sean escuchados por los extranjeros, dado que todo contacto se hace a través de los representantes. Así, la organización impuesta desde fuera tiene un efecto directo sobre el estatus de las mujeres a medida que sean más las críticas en lo referente a la supervivencia cultural que sean canalizadas a través de la ANAP, cuyos líderes son todos varones. Por otra parte, la ANAP combatela fuerte erosión del estatus de las mujeres que ha ocurridoen otras partes, por considerarlas como partícipes iguales en la organización e igualmente elegibles para recibir préstamos y asistencia.

Las mujeres continúan siendo las mayores productoras de alimentos, y la existencia de tierras de la comunidad repartidas de acuerdo con las familias excluye el que se les pueda definir como dependientes (como suele ser el caso bajo disposiciones estatales). Sin embargo, en la medida que el grupo recurre con mayor confianza al comercio externo y a las estructuras políticas que 
confieren a los varones un acceso mayor al estatus público (legal y oficialmente) y recursos de valor (dinero), las mujeres pierden la autonomía que tenían en el sistema tradicional así como pierden la capacidad de controlar los recursos valiosos (manufacturas tradicionales de las mujeres) y el prestigio asociado con ellos. El que los hombres, más que las mujeres, interactuen con la cultura dominante se refleja en el hecho de que aquellos Asháninka más próximos al mercado, muchos más hombres que mujeres hablan español (esto refleja también una educación diferenciada de niños y niñas). En sus interacciones con los extraños, las aludidas mujeres son más reticentes a hablar, y cuando hablan, responden por intermedio de sus esposos (u otros hombres). Este es un agudo contraste con la participación activa de las mujeres en las conversaciones de grupo en las comunidades tradicionales.

Finalmente, una de las primeras y más poderosas fuerzas de transformación de lo nativo alrededor del mundo han sido las misiones. Una influencia mayor hasta en las comunidades del área de estudio ha sido la misión adventista. En otras regiones las misiones cristianas han sido acusadas de fomentar un rol de subordinación para las mujeres mediante actividades organizativas y educativas y su concepción teológica del lugar de la mujer (Rogers 1980:36); si no es de otro modo lo cierto es que el rol modelo presentado por algunas de las áreas misioneras era firmemente patriarcal, promoviendo la subordinación y obediencia de las esposas a sus maridos. El rol de las misiones de disuación de los enfrentamientos, la poliginia y el consumo del masato, seguramente ha afectado las relaciones entre hombres y mujeres. La importancia del masato como producto de valor de las mujeres apreciado internamente entre los Asháninka (visto por los Stocks como un indicador del estatus de la mujer) debe aguardar una investigación ulterior. Su consumo, suprimido por muchos años por las misiones, goza de un renacimiento (después de haber sido expulsado por los misioneros americanos en los pasados años 70), especialmente como parte de los festivales tradicionales del grupo. Todavía es producido en forma cooperativa por varias o un grupo de mujeres, y (a diferencia de lo referido respecto a los Shipibo) la cerveza comercial todavía resulta demasiado costosa en estándares nativos como para convertirse en sustituto en un futuro cercano. Los cambios políticos a nivel nacional durante la década del 70 ocasionaron una dramática dismininución de la influencia de la Misión Adventista en la región, lo que dio origen a un retorno a los patrones tradicionales de las comunidades Asháninka y a sus prácticas dietéticas.

La expansión de la producción para efecto de incluir cosechas para la venta probablemente ha significado un aumento de las faenas agrícolas de las 
mujeres entre los Cocamilla, los Shipibo, 'y en un menor grado entre los Asháninka; sin embargo, en todas estas tres sociedades es el hombre quien controla los ingresos de la producción para el mercado. Puesto que el programa para el desarrollo de la selva tropical peruana apunta a la extracción de recursos, primordialmente productos agrícolas para alimentar al resto de la nación, y hacia el desarrollo de nuevos mercados de consumo (Smith 1982:80, Latin American Economic Report 1978:395) es improbable que los productos no agrícolas de las mujeres encuentren un lugar en el mercado nacional. De hecho, el incremento de la labor agrícola de las mujeres ha sido, entre los Asháninka por lo menos, temporalmente desplazado por la sustitución de manufacturas industriales, pero el punto en el que vuelva la disminución no puede estar lejano.

El cambio en la región está inextricablemente ligado a factores generados a niveles locales, nacionales e internacionales. La destrucción general de las selvas tropicales del mundo está en relación con las condiciones globales político económicas, y el tráfico internacional de narcóticos tiene efectos poderosos sobre la economía peruana, lo que a su vez incide en el desarrollo regional. Los programas políticos del Perú previos al gobiemo socialdemócrata mantuvieron protegidos a los indígenas, pero la situación económica desesperada causada por la descomunal deuda externa perjudicó la capacidad de implementarlos.

Durante la última década, el área del Valle del Pichis se ha visto agudamente afectada por la poderosa combinación de fuerzas del narcotráfico y la violencia de la insurgencia política. Los Asháninka sufrieron, pero superaron, un breve período de insurgencia y contrainsurgencia en los 60 conectado al movimiento del Che Guevara en Bolivia, pero no estu vo marcado por el nivel de violencia de los movimientos actuales ${ }^{(4)}$, ni por la tentación de dinero rápido proveniente de los comerciantes de cocaína. Estas mismas fuerzas han detenido, al menos por ahora, el influjo de los colonos, los planes para la reanudación del operar de la misión, y los intentos de desarrollo del gobierno.

Los eventos de los años recientes han conducido incluso a una incertidumbre mayor al observar el futuro de los Asháninka. En Diciembre de 1989 miembros del MRTA secuestraron al líder de la ANAP, Alejandro Calderón Espinoza, en un intento de coactarlo para que endosara su causa ${ }^{(5)}$. El insistió en la autonomía Asháninka y fue asesinado el 15 de Diciembre ${ }^{(6)}$. Su hijo mayor asumió su cargo. Los miembros de la ANAP, ultrajados, le declararon la guerra 
a los grupos subversivos y narcotráfico tomando el control de la capital del distrito, Puerto Bermudez el 26 de diciembre (Silvestre 1990:28). Subsecuentes reportes desde la región indican que las fuerzas armadas están colaborando con los Asháninka en su intento de restaurar la normalidad en el área. Ha habido esporádicos incidentes de violencia desde entonces, y muchos colonos han huido. La reafirmación de un real control territorial por parte de los indigenas no es de pequeña significación, pero será tratado en otra parte.

Más recientemente, la atención nacional e internacional se ha desviado hacia la problemática de los Asháninka de la región de Satipo-Ene-Perene donde una cantidad de 10,000 personas ha sido desplazada por la violencia política, y otros 5,000 son mantenidos prisioneros por grupos insurgentes (Rodríguez Vargas 1993:22; Miami Herald 6/21 1993; El Nuevo Herald 8/22 1993). Una solamente puede especular llegada a este punto sobre los efectos de una reemergencia de los enfrentamientos en el estatus de las mujeres ${ }^{(7)}$.

En el largo plazo, la presión sobre la base de recursos de las poblaciones indígenas de la selva amazónica, en el Perú como en otros lugares, inevitablemente ocurrirá si los planes nacionales de desarrollo queincluyen la colonización masiva son llevados a cabo. A medida que se incremente la colonización y haya menos tierra libre disponibles los patrones de vida indígenas cambiarán hacia formas familiares neolocales más nucleares. El resultado será un aumento del aislamiento doméstico de la mujer y la reducción del tiempo que pueda pasar conjuntamente con otras mujeres, fomentando la erosión de su base de au tonomía y aumentando su carga de trabajo individual. Debido al uso más intensivo de la tierra, declinarán la fertilidad y la productividad del suelo, y desherbar, el problema más difícil de la horticultura en la selva tropical durante la época de cultivo, se hará mucho más trabajoso y consumirá más tiempo. La subsistencia y la producción para el mercado demandarán cada vez más grandes esfuerzos a hombres y mujeres, y hará que tanto el trabajo jornalero masculino y la labor agrícola de las mujeres sean más necesarias y frecuentes. La evidencia emanada de otros estudios sugiere también que, salvo circunstancias verdaderamente inusuales, las mujeres perderán autonomía y estatus en relación a los hombres al mismo tiempo que se harán menos autosuficientes.

* Este artículo es una traducción del original en inglés presentado por la autora. El trabajo de traducción fue encargado al Sr. Sergio Ramirez Franco por el CAAAP. 


\section{NOTAS}

(1) Mucha literatura del pasado se refiere a estas personas como "Campa", un nombre impuesto por los europeos durante los primeros contactos. El nombre que ellos usan para referirse a sí mismos es Asháninka.

(2) Los datos concernientes a las diferencias hombre-mujer en cuanto a riqueza material no son válidos para las sociedades amazónicas cubiertas aquí. Aparentemente las fuentes de subsistencia todavia se hallan uniformemente disponibles y no hay patrones de diferencias de bienestar entre hombres y mujeres que pudiera ser medido en términos de nutrición, salud o cuidado de los niños.

(3) La Ley de Comunidades Nativas fue decretada en 1975. La identificación, agrimensuray titulación tuvo lugar hasta 1977 cuando fue interrumpida con aproximadamente el 30\% de los grupos titulados (Smith 1982). Fue resumida con estipulaciones menos favorables en 1981.

(4) El movimiento más extendido y más violento es Sendero Luminoso, pero el Movimiento Revolucionario Tupac A maru (MRTA) también está muy activo en la región Pichis. Ambos grupos han intentado sin éxito enlistar en sus filas al soporte de los Asháninka.

(5) Debido al incremento de la actividad guerrillera en el área' (y a un fuerte sentimiento en contra de cualquier intervención) las comunidades miembros de la ANAP establecieron una policía que recibe sólo a aquellos visitantes que portan una carta oficial de presentación de Calderón. El MRTA probablemente insistió en que él les otorgara un documento análogo, y él rehusó.

(6) El MRTA proclamó que su "ejecución" se debía a su participación en la captura del líder de la guerrilla en 1965.

(7) Algunos estudiosos sugieren que la existencia de los enfrentamientos tiene como correlato la subordinación femenina (Divale y Harris 1976), pero otros han notado un aumento de la importancia de las mujeres bajo algunas circunstancias (Rodriguez Vargas 1993: 33). 
Amazonía Peruana

\section{BIBLIOGRAFIA}

CHIRIF TIRADO, ALBERTO

1978 Notas sobre el Colonialismo Ecológico. En: Etnicidad y Ecología. Lima.

DENEVAN, WILLIAM

1971 Campa Subsistence in the Gran Pajonal, Eastern Peru. Geographical Review 61(4):496-518.

DIVALE, WILLIAM T. AND MARVIN HARRIS

1976 Population, Warfare, and the Male Supremacist Complex. American Anthropologist 78:521 - 38

EL NUEVO HERALD

1993 Unánime repulsa en Perú a matanzas de Asháninkas. 22 de agosto:3A, Miami.

HARRIS, MARVIN

1983 Cultural Anthropology. Harper \& Row, New York.

JOHNSON, ALLEN

1978 In Search of the Affluent Society. Human Nature, September: 50 - 59

JOHNSON, ORNA AND ALLEN JOHNSON

1975 Male-Female Relations and the Organization of Work in a Machiguenga Community. American Ethnologist 2(4): 634 - 48.

LATIN AMERICAN ECONOMIC REPORT 1978 6(1): 395

MURPHY, YOLANDA AND ROBERT MURPHY

1974 Women of the Forest. Columbia University Press, N.Y.

RODRÍGUEZ VARGAS, MARISOL

1993 Desplazados de la Selva Central: el caso de los asháninka. Documentos de Trabajo. CAAAP, Lima

ROGERS, BARBARA

1980 The Domestication of Women: Discrimination in Developing Societies. Tavistock Publications, London. 
SANDAY, PEGGY R.

1981 Female Power and Male Dominance: on the Origins of Sexual Inequality. Cambridge University Press, Cambridge.

SHAPIRO, JUDITH R.

1972 Sex Roles and Social Structure Among the Yanomama Indians of Northern Brazil. Ph. D. Dissertation, Columbia University, N.Y.

SHOSTAK, MARJORIE

1981 Nisa: the Life and Words of a Kung Woman. Harvard University Press, Cambribge.

SILVESTRE, MIGUEL

1990 "La Guerra Campa" Si 3 (151): 26 - 31. Lima, Perú.

SISKIND, JANET

1973 To Hunt in the Morning. Oxford University Press, N.Y.

SMITH, RICHARD CHASE

1982 The Dialectics of domination in Peru: Native Communities and the Myth of the Vast Amazonian Emptiness. Occasional Paper\#8,Cultural Survival, Cambridge, Massachusetts.

STOCKS, KATHLEEN AND ANTHONY STOCKS

1984 Status De La Mujer y Cambio Por Aculturación: Casos del Alto Amazonas. Amazonía Peruana 5(10): $65-77$.

THE MIAMI HERALD

1993 Indians reclaiming traditions. June 21:6A.

TIZON, JUDY

1975 The Campa Indians of Puerto Bermudez Peru: the People and the Land. ms: Ph.D. dissertation, U.C. Santa Barbara.

1988 Research Reporton Sabbatical Leave. University of Southern Maine. m.s. 